\title{
Langmuir
}

pubs.acs.org/Langmuir

(C) 2010 American Chemical Society

\section{Acidic pH-Responsive Nanogels as Smart Cargo Systems for the Simultaneous Loading and Release of Short Oligonucleotides and Magnetic Nanoparticles}

\author{
Smriti R. Deka,${ }^{\dagger}$ Alessandra Quarta,${ }^{\dagger}$ Riccardo Di Corato, ${ }^{\dagger}$ Andrea Falqui,${ }^{\dagger}$ Liberato Manna, \\ Roberto Cingolani, ${ }^{\dagger}$ and Teresa Pellegrino*, ${ }^{\dagger, \dagger}$ \\ ${ }^{\dagger}$ Istituto Italiano di Tecnologia, via Morego 30, 16163, Genova, Italy, and ${ }^{\star}$ National Nanotechnology \\ Laboratory of CNR-INFM and IIT Research Unit, via per Arnesano km 5, 73100, Lecce, Italy
}

Received February 1, 2010. Revised Manuscript Received March 1, 2010

\begin{abstract}
Smart materials able to sense environmental stimuli can be exploited as intelligent carrier systems. Acidic pHresponsive polymers, for instance, exhibit a variation in the ionization state upon lowering the $\mathrm{pH}$, which leads to their swelling. The different permeability of these polymers as a function of the $\mathrm{pH}$ could be exploited for the incorporation and subsequent release of previously trapped payload molecules/nanoparticles. We provide here a proof of concept of a novel use of $\mathrm{pH}$-responsive polymer nanostructures based on 2-vinylpyridine and divinylbenzene, having an overall size below $200 \mathrm{~nm}$, as cargo system for magnetic nanoparticles, for oligonucleotide sequences, as well as for their simultaneous loading and controlled release mediated by the $\mathrm{pH}$.
\end{abstract}

\section{Introduction}

Research on nanocomposites aims at developing and miniaturizing structures made of different functional entities, each of them able to carry out specific tasks. In order to design multifunctional nanostructures that might serve as new medical devices, it is crucial to identify "smart materials" that are capable of responding to defined stimuli. Hydrogels are an interesting class of functional materials that have been exploited as intelligent cargo systems for the encapsulation and the delivery of different active molecules, such as oligonucleotides or anticancer drugs, ${ }^{1-4}$ and that can be useful for the capture and for the controlled release of inorganic nanoparticles. These polymers, whose structure is composed of a three-dimensional network of cross-linked units, can undergo substantial modifications of some of their properties (such as their total charge or their hydrophobicity/ hydrophilicity balance) as a consequence of small variations in the local environment, like a change in $\mathrm{pH}$ or in the temperature.,

Hydrogels in their bulk form have been applied so far in implants, contact lenses, dental materials, and vascular grafts. ${ }^{1,7}$ In past years, there has been increasing research activity focused on the miniaturization of hydrogel particles (henceforward referred to as "nanogels") and on the study of their potential as drug delivery agents. ${ }^{1}$ Research in this area has shown that in order for nanogels to have reliable structure-properties relationships one needs to finely control both their size and their purity. ${ }^{6}$ Size control is particularly critical because a nanogel designed for in vivo delivery of drugs, genes, or nanoparticles should be smaller

*Corresponding author. teresa.pellegrino@unile.it; tel. +39 0832298 214; fax +390832298230 .

(1) Schmaljohann, D. Adv. Drug Delivery Rev. 2006, 58, 1655.

(2) Nayak, S.; Lyon, L. A. Angew. Chem.-Int. Ed. 2005, 44, 7686.

(3) Gupta, P.; Vermani, K.; Garg, S. Drug Discovery Today 2002, 7, 569

(4) Vinogradov, S. V.; Bronich, T. K.; Kabanov, A. V. Adv. Drug Delivery Rev. 2002, 54,135 .

(5) Ganta, S.; Devalapally, H.; Shahiwala, A.; Amiji, M. J. Controlled Release 2008, 126, 187.

(6) Raemdonck, K.; Demeester, J.; De Smedt, S. Soft Matter 2009, 5, 707

(7) Liu, S.; Maheshwari, R.; Kiick, K. L. Macromolecules 2009, 42, 3.

(8) Takakura, Y.; Mahato, R. I.; Hashida, M. Adv. Drug Delivery Rev. 1998, 34,93 . than $200 \mathrm{~nm}^{8-13}$ Once injected intravenously, a nanogel smaller than this size will remain colloidally stable, it will not be sequestered immediately by the reticulo-endothelial system, ${ }^{11}$ and hence, it will stay in circulation for a sufficiently long time to reach the tumor regions and pass through the pore vessels at these regions. ${ }^{1,9,14}$

While nanogels based on temperature-responsive polymers are generally designed to be altered by external stimuli, those based on $\mathrm{pH}$-responsive polymers can respond to variations in the intracellular or tissue environment. ${ }^{1}$ It is known, for example, that certain cancer tissues, due to hypoxia environment, exhibit an extracellular $\mathrm{pH}$ around $6.5,{ }^{15}$ while the $\mathrm{pH}$ of some intracellular compartments, like lysosomes, is around 4.5. ${ }^{16}$ The $\mathrm{pH}$-dependent swelling behavior of a nanogel can be useful not only for the release of the cargo, but also for its loading. Indeed, when the nanogel swells, its permeability increases, allowing either for the incorporation of molecules/nanoparticles or alternatively for the release of previously trapped payloads. So far, several $\mathrm{pH}$-responsive polymers have been widely used as a controlled drug delivery system. ${ }^{17-21}$ In some studies, $\mathrm{pH}$-responsive polymers have been exploited as templates for the in situ synthesis of nanoparticles, and the resulting hybrid systems were tested mainly in catalytic applications. ${ }^{22,23}$ Only in a few works were $\mathrm{pH}$-responsive

(9) Mitragotri, S.; Lahann, J. Nat. Mater. 2009, 8, 15.

(10) Decuzzi, P.; Pasqualini, R.; Arap, W.; Ferrari, M. Pharm. Res. 2009, 26, 235.

(11) Cairns, R.; Papandreou, I.; Denko, N. Mol. Cancer Res. 2006, 4, 61

(12) Torchilin, V. P. Adv. Drug Delivery Rev. 2006, 58, 1532

(13) Torchilin, V. P. J. Controlled Release 2001, 73, 137.

(14) Prokop, A.; Davidson, J. M. J. Pharm. Sci. 2008, 97, 3518.

(15) Gerweck, L. E.; Seetharaman, K. Cancer Res. 1996, 56, 1194.

(16) Grabe, M.; Oster, G. J. Gen. Physiol. 2001, 117, 329.

(17) Dai, S.; Ravi, P.; Tam, K. C. Soft Matter 2008, 4, 435.

(18) Wu, D. Q.; Sun, Y. X.; Xu, X. D.; Cheng, S. X.; Zhang, X. Z.; Zhuo, R. X. Biomacromolecules 2008, 9, 1155

(19) Zhao, C.; Zhuang, X.; He, P.; Xiao, C.; He, C.; Sun, J.; Chen, X.; Jing, X. Polymer 2009, 50, 4308 .

(20) Qu, T.; Wang, A.; Yuan, J.; Gao, Q. J. Colloid Interface Sci. 2009, 336, 865.

(21) Peppas, N. A.; Hilt, J. Z.; Khademhosseini, A.; Langer, R. Adv. Mater. 2006, $18,1345$.

(22) Zhang, J. G.; Xu, S. Q.; Kumacheva, E. J. Am. Chem. Soc. 2004, 126, 7908

(23) Palioura, D.; Armes, S. P.; Anastasiadis, S. H.; Vamvakaki, M. Langmuir 2007, 23, 5761 . 
polymers combined with magnetic nanoparticles, ${ }^{24,25}$ and in such cases, the magnetic nanoparticles were always covalently linked to the nanogel networks. In most of those works, the magnetic nanoparticles were actually used as templates on which the polymer was grown around, ${ }^{25,26}$ or vice versa, the polymer was used as template on which the magnetic nanoparticles were nucleated (and remained bound to it). To our knowledge, there has been no report so far on the use of nanogels as carrier systems for the controlled release of magnetic nanoparticles.

Among the vast class of nanoparticles, iron oxide nanoparticles (IONPs, both maghemite and magnetite) are superparamagnetic nanocrystals that have been widely investigated as drug delivery, diagnosis, and therapeutic agents. ${ }^{27}$ Due to their intrinsic magnetic properties, IONPs are ideal candidates as delivery agents: when exposed to an external magnetic field of moderate intensity, they are able to accumulate to the site where the magnet is positioned, while upon removal of the magnet, they do not undergo aggregation, as they do not exhibit any residual magnetization. Furthermore, IONPs are valuable contrast agents in magnetic resonance imaging (MRI) because their magnetic moments can affect the relaxivity of the water molecule protons present in the tissues, resulting in a negative contrast in the area where the nanoparticles are localized. ${ }^{28}$ IONPs can also serve as colloidal mediators for generating heat for hyperthermia treatment in cancer therapy, under the application of appropriate alternating magnetic fields. ${ }^{29}$ The inclusion of IONPs in the nanogel confers to it all the additional advantages of IONPs as described above.

In the present work, we employ acidic $\mathrm{pH}$-responsive nanogels as delivery systems for different types of payloads, namely, IONPs and short oligonucleotide sequences, and combinations of them. We have modified a previously reported synthetic procedure for preparing $\mathrm{pH}$-responsive nanogels $\mathrm{s}^{30}$ in order to obtain nanogels with sizes tunable from 40 to $200 \mathrm{~nm}$, and we have tested those materials as carrier systems. A full characterization based on transmission electron microscopy (TEM), photoluminescence spectroscopy, confocal microscopy, and dynamic light scattering (DLS) was carried out in order to elucidate the loading and the release processes of short DNA sequences, of IONPs, and the combined loading and release of both payloads at the same time. Our $\mathrm{pH}$ and of salt concentration results show that full control over the loading and the release of IONPs and DNA is clearly achieved.

\section{Experimental Section}

2.1. Chemicals. All chemicals were of the highest purity available and were used as received. 2-Vinyl pyridine (2-VP, 97\%), sodium tetraborate decahydrate, boric acid, as well as all the disposable materials were purchased from Sigma-Aldrich. Divinylbenzene (DVB), 2,2'-azobis(2-methylpropionamidine)dihydrochloride (AIBA), and Diamine-PEG (MW 897) were purchased from Fluka. The HPLC purified oligonucleotide sequence modified at the $5^{\prime}$ end with Cy3 (5'-CAC CAC ACG GTC GGC AGC CAC GGT A-3', henceforth referred to as Cy3-DNA) was purchased from Thermo Electron Corporation. Doubly distilled deionized water $(\mathrm{pH} \sim 6$ ) was used for the polymerization

(24) Bhattacharya, S.; Eckert, F.; Boyko, V.; Pich, A. Small 2007, 3, 650.

(25) Zhou, L. L.; Yuan, J. Y.; Yuan, W. Z.; Sui, X. F.; Wu, S. Z.; Li, Z. L.; Shen, D. Z. J. Magn. Magn. Mater. 2009, 321, 2799.

(26) Zhou, L. L.; Yuan, J. Y.; Yuan, W. Z.; Zhou, M.; Wu, S. Z.; Li, Z. L.; Xing, X. H.; Shen, D. Z. Mater. Lett. 2009, 63, 1567.

(27) Laurent, S.; Forge, D.; Port, M.; Roch, A.; Robic, C.; Elst, L. V.; Muller, R. N. Chem. Rev. 2008, 108, 2064

(28) Na, H. B.; Song, I. C.; Hyeon, T. Adv. Mater. 2009, 21, 2133.

(29) Gazeau, F.; Levy, M.; Wilhelm, C. Nanomedicine 2008, 3, 831.

(30) Dupin, D.; Fujii, S.; Armes, S. P.; Reeve, P.; Baxter, S. M. Langmuir 2006, 22,3381 . and for all experiments. Poly(maleic anhydride-alt-1-tetradecene) (PC 14) was purchased from Sigma-Aldrich, although at present, this polymer is no longer commercially available. The reader can refer to a new polymer coating procedure implemented by us which employs a similar polymer, which is still commercially available. $^{3}$

2.2. Synthesis of Nanogels via Emulsion Polymerization. A series of polyvinyl pyridine nanogels were synthesized with a control over the size diameter of the nanogel below $200 \mathrm{~nm}$, following a procedure published by Dupin et al. ${ }^{30}$ and modified by us. As an example, we describe here the experimental conditions for the synthesis of nanogels of about $110 \mathrm{~nm}$ in diameter (as determined by transmission electron microscopy, TEM). A mixture of 2-vinyl pyridine (2-VP, $0.25 \mathrm{~g}$ ) and divinylbenzene (DVB, $0.013 \mathrm{~g}$ ) was dissolved in $60 \mathrm{~mL}$ of water in a round-bottom flask. The $\mathrm{pH}$ of the resulting solution was 8.3 immediately after mixing. The flask was sealed with a rubber septum, and the aqueous solution was degassed at ambient temperature by five vacuum/ nitrogen cycles. The degassed solution was constantly stirred with a magnetic stirrer and heated at $60 \pm 1{ }^{\circ} \mathrm{C}$. After $20 \mathrm{~min}$, the solution of the AIBA initiator $(0.022 \mathrm{~g}$ in $1 \mathrm{~mL}$ water $)$ was added to the flask, and after $15 \mathrm{~min}$, the solution in the flask turned milky white, indicating the nucleation of the nanogels. This solution was left to polymerize for a further $2 \mathrm{~h}$ under stirring conditions at $60{ }^{\circ} \mathrm{C}$, after which the flask was opened to air in order to expel the nitrogen atmosphere and to stop the reaction. In order to remove the residual monomers in solution, the 2-VP nanogel particles were washed at least 10 times with a Millipore Dialysis System (MWCO 100.000) on centricone tubes, and the reaction mixture was centrifuged at $4000 \mathrm{rpm}$ for $30 \mathrm{~min}$. Fresh water was added each time before any centrifugation. All dispersions were diluted using Milli-Q water $(18.2 \mathrm{M} \Omega)$ that had been ultrafiltered $(0.20 \mu \mathrm{m}$ filter) prior to use. The solution $\mathrm{pH}$ was adjusted by using a solution of $\mathrm{HCl}(0.1 \mathrm{M})$ or $\mathrm{NaOH}(0.1 \mathrm{M})$ and the $\mathrm{pH}$ was checked with a $\mathrm{pH}$-meter equipped with a microelectrode (Crison $\mathrm{pH}$ Meter Basic 20+). In order to tune the size of the nanogels below $200 \mathrm{~nm}$ in diameter, we have varied the molar ratio of 2-VP/DVB by changing the amount of 2-VP added, while keeping constant all the other reaction conditions, as described above (Table 1).

\subsection{Preparation of Diamino-PEG Conjugated Iron Oxide} Nanocrystals. Iron oxide nanocrystals (diameter of $7 \mathrm{~nm}$ ) were synthesized according to the Sun method. ${ }^{32}$ The "as synthesized" nanoparticles had a capping of oleic acid and oleylamine and were soluble in organic solvents. They were transferred into water by using a polymer coating procedure developed by us. ${ }^{33}$ Briefly, the nanoparticles were wrapped in an amphiphilic polymer shell made of poly(maleic anhydride alt-1-tetradecene), and such shell was then cross-linked using a triamine. The nanocrystals were therefore soluble in water and were negatively charged, as determined by zeta potential measurements (Table 1, Supporting Information), due to the outstretched carboxylate moieties of the polymer molecules. In order to remove the excess free polymer, an ultracentrifugation step was performed at $150000 \mathrm{rcf}$ on a continuous sucrose gradient. ${ }^{31}$ Then, diamino-PEG molecules (molecular weight $897 \mathrm{Da}$ ) were bound to the carboxy groups at the nanoparticle surface via EDC chemistry. The amino-PEG molecules were introduced in order to make the nanoparticles more stable at different conditions of $\mathrm{pH}$ and ionic strength. ${ }^{34}$ In detail, to $500 \mu \mathrm{L}$ of a nanocrystal solution $6 \mu \mathrm{M}, 500 \mu \mathrm{L}$ of a solution containing a molar ratio of diamino-PEG/NP equal to 500 were added, and after mixing, $500 \mu \mathrm{L}$ of a solution containing an excess molar ratio of EDC/NP (equal to 75000) was also

(31) Di Corato, R.; Quarta, A.; Piacenza, P.; Ragusa, A.; Figuerola, A.; Buonsanti, R.; Cingolani, R.; Manna, L.; Pellegrino, T. J. Mater. Chem. 2008, $18,1991$.

(32) Sun, S. H.; Zeng, H. J. Am. Chem. Soc. 2002, 124, 8204

(33) Pellegrino, T.; Manna, L.; Kudera, S.; Liedl, T.; Koktysh, D.; Rogach, A. L.; Keller, S.; Radler, J.; Natile, G.; Parak, W. J. Nano Lett. 2004, 4, 703.

(34) Sperling, R. A.; Pellegrino, T.; Li, J. K.; Chang, W. H.; Parak, W. J. $A d v$. Funct. Mater. 2006, 16, 943. 
Table 1. Experimental Conditions for the Synthesis of Nanogels of Different Diameters ${ }^{a}$

\begin{tabular}{lcccccc}
\hline sample name & 2-VP $(\mathrm{g})$ & DVB $(\mathrm{g})$ & {$[2-\mathrm{VP}] /[\mathrm{DVB}]$ Molar ratio } & TEM diameter $(\mathrm{nm})$ & DLS diameter $(\mathrm{nm})$ & polydispersity index \\
\hline NG197 & 0.754 & 0.013 & 71.6 & $197 \pm 10$ & $223 \pm 60$ & 0.073 \\
NG142 & 0.505 & 0.013 & 48.3 & $142 \pm 7$ & $185 \pm 51$ & 0.035 \\
NG110 & 0.250 & 0.013 & 23.7 & $110 \pm 8$ & $137 \pm 29$ & 0.028 \\
NG75 & 0.101 & 0.013 & 9.5 & $75 \pm 7$ & $92 \pm 20$ & 0.022
\end{tabular}

${ }^{a}$ By varying the molar ratio between the 2-VP and the DVB (column 4), while keeping all the other reaction condition constant, it was possible to tune the sizes of the nanogels from $41 \mathrm{~nm}$ to 197, as determined by statistical TEM measurements (column 5) on an average of 100 nanogel particles. The hydrodynamic diameters of the same samples, as measured by dynamic light scattering, (column 6) were clearly bigger. The low polydispersity index indicates uniform size distribution (column 7) (all measurements were conducted at $\mathrm{pH}$ 7.5).

added. After a reaction time of $3 \mathrm{~h}$ at room temperature under vigorous stirring, the unbound diamino-PEG molecules were removed by performing several washing steps on centrifuge tubes having a MWCO of 30000 .

2.4. Loading and Release Experiments of Diamino-PEG Conjugated Iron Oxide Nanocrystals in the Nanogel. The loading of IONPs in the nanogels was performed as follows: $3 \mathrm{~mL}$ of a solution of nanogel in water $(0.053 \mathrm{w} / \mathrm{v}(\mathrm{g} / \mathrm{mL}) \%)$ were mixed with $9 \mu \mathrm{L}$ of a solution of PEG-coated $\gamma-\mathrm{Fe}_{2} \mathrm{O}_{3}$ (the concentration of nanoparticles in this solution was equal to $14.5 \mu \mathrm{M})$ and the resulting mixture was stirred for $24 \mathrm{~h}$ at $\mathrm{pH} 3.5$ at room temperature. Under these conditions, the swollen nanogels started incorporating the IONPs. The $\mathrm{pH}$ of the solution was then increased slowly to 7 by dropwise addition of a solution of $\mathrm{NaOH} 0.1 \mathrm{M}$ (a slight turbidity appeared as soon as the $\mathrm{pH}$ reached 5.25, indicating shrinkage of the nanogels). Soon after, the nanogels loaded with IONPs were separated from free excess of IONPs using a magnet: the solution was placed close to the magnet, and within $1 \mathrm{~h}$, the nanogels loaded with nanoparticles were attracted toward it. The nanogels were characterized by TEM and by DLS measurements. For the release experiments, the $\mathrm{pH}$ of the nanogel solution was decreased again to 3.5 by dropwise addition of a solution of $\mathrm{HCl}(0.1 \mathrm{M})$, in order for the nanogels to swell again. For the quantification of the average number of IONPs loaded within the nanogel, the determination of iron concentration was carried out via elemental analysis on the loaded IONPsnanogels (as explained more in detail in the paragraph of the 2.11).

2.5. Loading Experiment of Cy3-DNA in Nanogel and Subsequent Release. For the loading experiments of oligonucleotide sequences of 25 bases, $3 \mathrm{~mL}$ of nanogel solution $(0.053$ $\mathrm{w} / \mathrm{v}(\mathrm{g} / \mathrm{mL} \%)$ were mixed with $9 \mu \mathrm{L}(100 \mathrm{pmol} / \mu \mathrm{L})$ of Cy3-DNA solution and the $\mathrm{pH}$ was adjusted to 3.5 by addition of $\mathrm{HCl} 0.1 \mathrm{M}$. The sample was left to stir for $24 \mathrm{~h}$ at room temperature, and soon after, the $\mathrm{pH}$ was increased again to 7 by dropwise addition of a solution of $\mathrm{NaOH} 0.1 \mathrm{M}$, after which it was left to stir for additional $3 \mathrm{~h}$. To remove the excess of free Cy3-DNA, the final solution was centrifuged on $100000 \mathrm{MWCO}$ amicon tubes at $3000 \mathrm{rpm}$. The process was repeated until all the free Cy3-DNA was washed away, as monitored by PL spectra on the filtered solution (5 to 7 washing steps on centricone tube of $4 \mathrm{~mL}$ were usually required). The free Cy3-DNA solution was collected on the lower part of the centricone tube, while the Cy3-DNA/nanogels were recovered on the upper side of the filter and were redispersed in $1 \mathrm{~mL}$ of water.

For the quantification of Cy3-DNA loaded within the nanogel, we have recorded the PL of loaded nanogel samples (both Cy3DNA/nanogel and Cy3-DNA-IONP/nanogel). We have then extrapolated the Cy3-DNA concentration of those samples on calibration curves of photoluminescence vs Cy3-DNA concentration (PL/Cy3-DNA concentration). These were obtained by preparing standard solutions at known Cy3-DNA concentrations, in which we have simulated the matrix. In more detail, for building the calibration curve for the Cy3-DNA/nanogel sample, to each of the standards at different DNA concentrations we have added the same amount of nanogel that we had in our sample. Likewise, in order to build the calibration curve for the Cy3DNA-IONP/nanogel sample, to each of the standards we have added an amount of nanogel and IONP at the same concentration that we had in our sample (Figure 8S, Supporting Information).
For the release experiment of the Cy3-DNA, $50 \mu \mathrm{L}$ of a solution of $\mathrm{NaCL}(5 \mathrm{M})$ were added to $1 \mathrm{~mL}$ of the above nanogel solution loaded with Cy3-DNA, and the $\mathrm{pH}$ was adjusted to 3.5. The sample was left under stirring for $72 \mathrm{~h}$, and soon after the solution was centrifuged on an amicon tube of MWCO 100000. PL spectra were recorded on fractions collected both on the upper part and on the lower part of the membrane.

2.6. Simultaneous Loading in Nanogel of Both Cy3-DNA and IONPs and Subsequent Release Experiments. To load IONPs and Cy3-DNA simultaneously within the nanogels, the same procedure as described above (to load IONPs and Cy3DNA separately) was applied. The only difference in the present case was that $3 \mathrm{~mL}$ of nanogel in water $(0.053 \mathrm{w} / \mathrm{v}(\mathrm{g} / \mathrm{mL} \%)$ was mixed together with $9 \mu \mathrm{L}$ of the Cy3-DNA solution (100 picomol/ $\mu \mathrm{L})$ and with $9 \mu \mathrm{L}$ of the IONPs solution $(14.5 \mu \mathrm{M})$, after which the $\mathrm{pH}$ was adjusted to $3.5 \mathrm{using} \mathrm{HCl} 0.1 \mathrm{M}$. Also, in this case the loading and the release were monitored by TEM and by PL.

2.7. Dynamic Light Scattering. Zeta potential and dynamic light scattering measurements (DLS) were performed on a Zetasizer Nano ZS90 (Malvern, USA) equipped with a $4.0 \mathrm{~mW}$ $\mathrm{He}-\mathrm{Ne}$ laser operating at $633 \mathrm{~nm}$ and with an avalanche photodiode detector. Measurements were made at $25^{\circ} \mathrm{C}$ in water. All the samples were filtered before analysis. $0.2 \mu \mathrm{m}$ filters were used for the nanogel alone, while for nanogel samples loaded with nanoparticles and Cy3-DNA solution, $0.5 \mu \mathrm{m}$ filters were preferred.

2.8. UV-vis Absorption, Photoluminescence (PL) Spectroscopy. UV-visible absorption spectra were measured using a Varian Cary 300 UV - vis spectrophotometer. Photoluminescence (PL) spectra were recorded on a Cary Eclipse spectrophotometer. To record the PL spectra of Cy3-DNA alone and in nanogel, the samples were excited at $500 \mathrm{~nm}$.

2.9. Transmission Electron Microscopy. TEM images were recorded on a JEOL jem 1011 microscope operated at an accelerating voltage of $100 \mathrm{kV}$. TEM samples were prepared by dropping a dilute solution of nanogel in water on carbon-coated copper grids and letting the solvent evaporate. The reported TEM diameters were measured on an average of 100 particles.

2.10. Confocal Microscopy Imaging. Confocal microscopy images were acquired with an Olympus FV-1000 microscope, equipped with an argon laser source (488 $\mathrm{nm}$ excitation line) and a DM488/405 type dichroic filter. The fluorescence reading channel was set at $565 \pm 25 \mathrm{~nm}$.

2.11. Elemental Analysis. An inductively coupled plasma atomic emission spectrometer (ICP-AES) Varian Vista AX was used to measure the concentration of $\mathrm{Fe}$ and thus the concentration of IONPs. The samples were digested in the following way: they were dissolved in a concentrated acid solution $(\mathrm{HCl} / \mathrm{HNO} 3$ $(3 / 1 \mathrm{v} / \mathrm{v})$ and were left for $24 \mathrm{~h}$, before performing elemental analysis. The Fe concentration was converted into nanoparticle concentration using a procedure described by us in a previously published paper. ${ }^{35}$ In detail, the average diameter of the nanoparticles was assessed via statistical analysis on TEM images. The average number of $\mathrm{Fe}$ atoms per nanoparticle was determined by

(35) Deka, S.; Quarta, A.; Lupo, M. G.; Falqui, A.; Boninelli, S.; Giannini, C.; Morello, G.; De Giorgi, M.; Lanzani, G.; Spinella, C.; Cingolani, R.; Pellegrino, T.; Manna, L. J. Am. Chem. Soc. 2009, 131, 2948. 

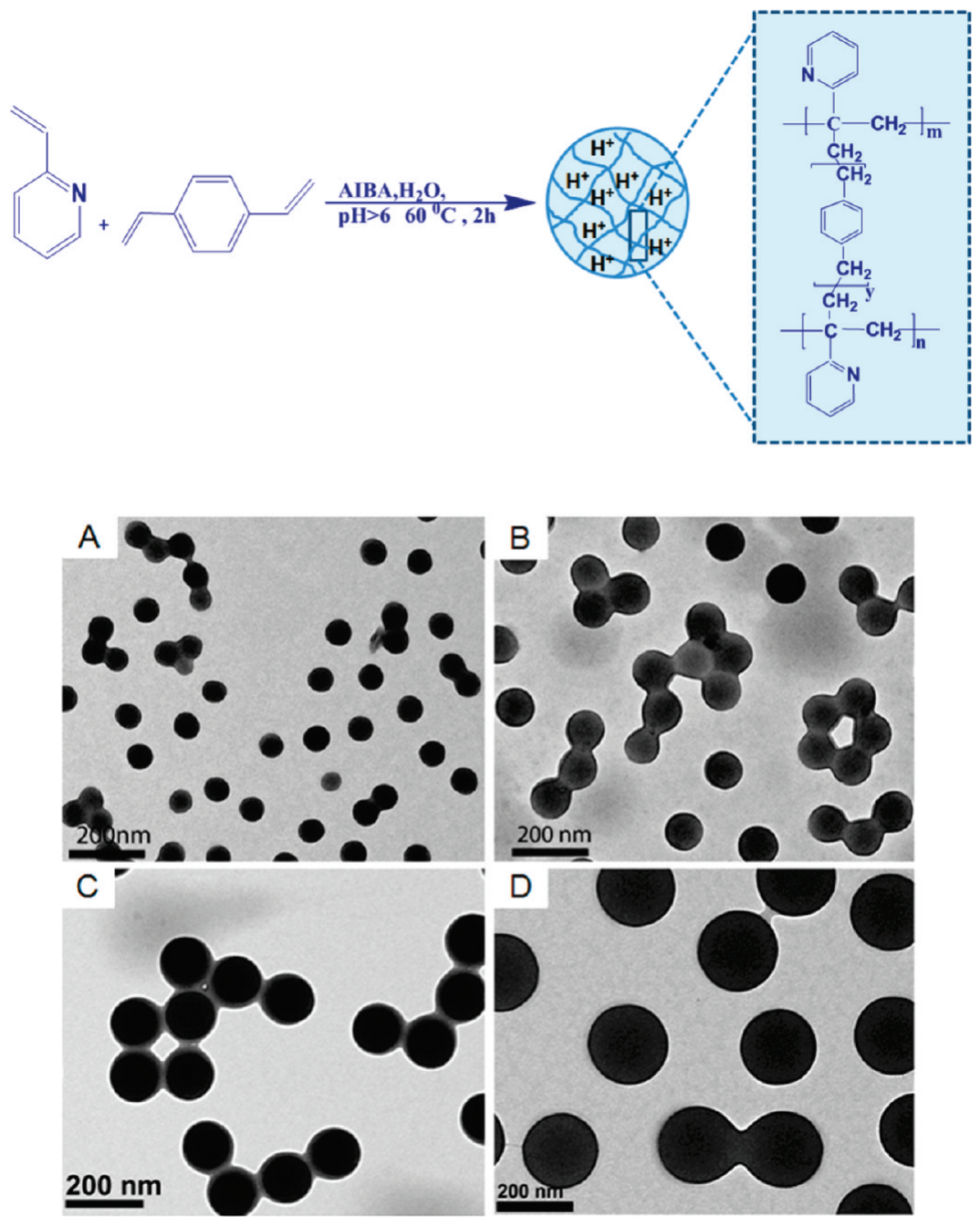

Figure 1. Sketch of the structure of the vinyl pyridine (VP) and divinylbenzene (DVB) units, which were employed for the synthesis of pH-responsive nanogels. TEM images of four different nanogel samples, with average diameters corresponding to (a) 75, (b) 110, (c) 142, and (d) $197 \mathrm{~nm}$ (the TEM diameters reported were estimated on an average of 100 nanogel particles; see Table 1, column 5).

building a structural model of the nanoparticle, with the same geometrical parameters of the nanoparticles as determined by TEM. Then, by knowing the average number of Fe atoms per nanoparticle and the total concentration of this species in solution, it is possible to determine the concentration of nanoparticles. In order to elucidate $\mathrm{Fe}^{3+}$ leakage in the condition of the loading experiment, we kept the IONPs at pH 3.5 overnight and we collected the supernatant solution, i.e., the solution separated from the IONPs by filtration on centricone filter. Finally, we measured the $\mathrm{Fe}$ concentration in both fractions.

\section{Results and Discussion}

3.1. Preparation of $\mathbf{p H}-\mathrm{Responsive} \mathrm{Nanogels} \mathrm{and} \mathrm{Char-}$ acterization of Their Swelling Behavior. The $\mathrm{pH}$-responsive nanogels employed in this study are based on copolymers of divinylbenzene (DVB) and vinyl pyridine (VP) (sketch of Figure 1). They were synthesized following earlier reported procedures, with minor modifications. ${ }^{30,36}$ This type of surfactant-free emulsion polymerization procedure was first described by Loxley and Vincent, ${ }^{36}$ who synthesized monodisperse cationic nanogels of 2-vinylpyridine by varying the amount of styrene (the hydrophobic monomer) and that of DVB (the cross-linker agent). The authors demonstrated a tight control over the particle size in the

(36) Loxley, A.; Vincent, B. Colloid Polym. Sci. 1997, 275, 1108. range between 160 and $200 \mathrm{~nm}$. According to a modified version of the Loxley and Vincent procedure, Dupin et al. ${ }^{30}$ have reported the synthesis of sterically stabilized PVP latexes at much higher solid density, and with control over the diameter from 300 to $1000 \mathrm{~nm}$. They used suitable stabilizer molecules, namely, monomethoxy-capped poly(ethylene glycol) methacrylate (PEGMA), and surfactant molecules named " 336 ".

Our interest in the present study was to control the size of the nanogels below $200 \mathrm{~nm}$, which is a more suitable size range for the potential use of such nanogels as cargo system, as highlighted above. We were able to synthesize a series of nanogels in the size range between 40 and $200 \mathrm{~nm}$, by reducing the monomer concentration of 2-VP, while keeping all the other parameters constant (Table 1 and Figure 1). Reducing the concentration of 2-VP corresponds to a decrease in the 2-VP/DVB molar ratio, or the same to an increase in the amount of DVB (the cross-linker monomer) per nanogel. The formation of smaller nanogels can be ascribed therefore to a higher degree of reticulation of the nanogel network. The smallest nanogels that we could prepare had a TEM diameter of about $40 \mathrm{~nm}$ (Figure 1S, Supporting Information).

For a given nanogel sample, the average diameter, as measured by DLS (Table 1, column 5), was slightly higher than that measured by TEM. This was expected, since the DLS measurements were carried out on hydrated nanogels. Moreover, the DLS 


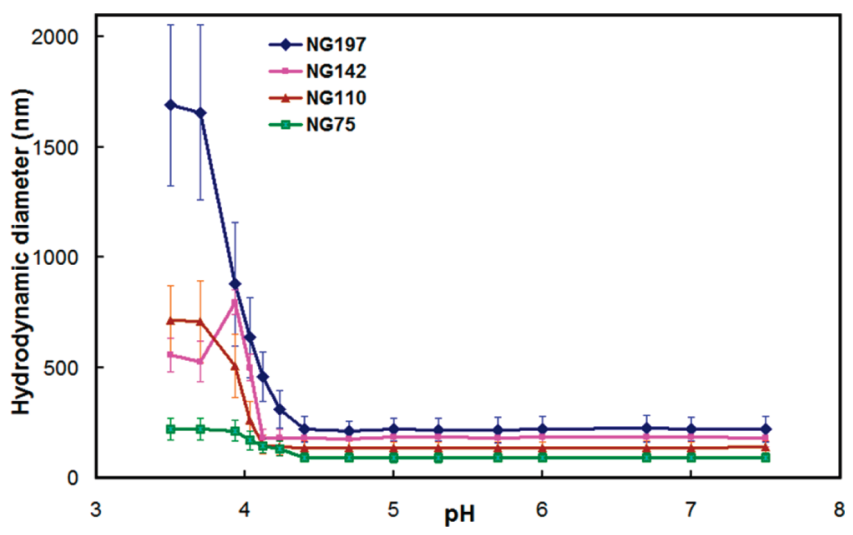

Figure 2. Swelling behavior of the nanogels. The DLS diameter is reported as a function of the $\mathrm{pH}$ (each measurement was carried out three times.) All nanogel samples exhibited a sharp swelling behavior below $\mathrm{pH} 4.3$.

polydispersity indexes were low, confirming the uniformity in size distribution of the nanogels (Table 1, column 7).

It is also worth highlighting that in our preparation we ran the reaction for $2 \mathrm{~h}$, while in previously reported methods the reaction time was $24 \mathrm{~h} .{ }^{30} \mathrm{We}$ additionally observed that by increasing the reaction time from 2 to $24 \mathrm{~h}$ the size of the nanogel increased, but the final nanogel was mostly aggregated (Figure $2 \mathrm{~S}$, Supporting Information).

To investigate the swelling behavior of the various nanogel samples in water, the $\mathrm{pH}$ of each nanogel solution was lowered from an initial value of 7.5 by dropwise additions of an $\mathrm{HCl}$ solution. After an equilibration time of about $10 \mathrm{~min}$, the nanogel diameters increased sharply at $\mathrm{pH}$ below 4.3 , due to progressive protonation of the nitrogen of the 2-vinylpyridine residues ${ }^{37}$ (Figure 2). Regardless of the starting size of the nanogels, the swelling occurred always at $\mathrm{pH}$ below 4.3. The various samples exhibited critical swelling transition at $\mathrm{pH}$ values between 4.3 and 3.9, depending on the ratio between 2-VP and DVB employed in the preparation of the nanogels (i.e., the degree of cross-linking of DVB).

The majority of the pyridine groups became protonated below $\mathrm{pH} 3.8$, and the average diameters of the nanogels reached a maximum due to the electrostatic repulsions between the strongly cationic chains.

Above $\mathrm{pH} 4.5$, the particles were in the swollen state, because of the absence of inner charges, and they behaved like conventional polymer latex particles. ${ }^{37}$ The swelling of the nanogel particles was also confirmed by visual inspection, as the solution turned from turbid, milky-white to clear when the $\mathrm{pH}$ was decreased from 7 to 3.5 (Figure 3S, Supporting Information). Zeta potential measurements indicated a strong cationic character of the nanogels at $\mathrm{pH} 3.5$, which is the $\mathrm{pH}$ at which the payload was usually loaded. However, even at $\mathrm{pH} 7$ the nanogels retained a slightly positive charge (Table 2, Supporting Information).

3.2. Loading and Release Experiments of Iron Oxide Nanoparticles. For the loading and release experiments, a nanogel sample having average "TEM" diameter equal to $110 \mathrm{~nm}$ at pH 7 was employed (henceforward referred to as "NG110"), and the loading and release process of IONPs was monitored by TEM (Figure 3). After mixing the nanogels with IONPs (PEG-coated nanoparticles, $7 \mathrm{~nm}$ in diameter) ${ }^{32-34}$ and upon switching the $\mathrm{pH}$ from 7 to 3.5 , the nanogel was turned into a swollen state (under

(37) Fernandez-Nieves, A.; Fernandez-Barbero, A.; Vincent, B.; de las Nieves, F. J. Macromolecules 2000, 33, 2114.
TEM, the edge of the nanogel was not sharply defined anymore; see Figure 3B). A gentle overnight shaking at room temperature was then followed by restoration of the solution $\mathrm{pH}$ back to 7 (by addition of $\mathrm{NaOH}$ ), which induced the shrinkage of the nanogels, inside which the IONPs remained entrapped (Figure 3C).

By application of a magnet, the nanogels loaded with IONPs could be recovered and they were separated by the excess of free IONPs (Figure 3D), as the former were attracted faster than the latter to the magnet (Figure 3D). In order to achieve a complete cleaning of the loaded nanogel from the free IONPs, a second purification step on Sephadex column was performed. The incorporation of the IONPs in the nanogel induced an appreciable increase of the average nanogel diameter, as determined by TEM (in one sample, for instance, it varied from $110 \pm 8 \mathrm{~nm}$ to $117 \pm 12 \mathrm{~nm}$ ).

DLS was additionally exploited to examine the behavior of the nanogels at each step of the procedure. Immediately after mixing the IONPs with the nanogel, at $\mathrm{pH} 3.5$ the DLS diameter of the nanogel was around $480 \pm 94 \mathrm{~nm}$ (Table 1, Supporting Information), which was lower than that of the nanogels when they were swollen at the same $\mathrm{pH}$ but in the absence of IONPs $(713 \pm$ $158 \mathrm{~nm}$ ). This reduced swelling of the nanogels might be due to the ionic interactions in solution between the IONPs and the nanogels. After switching the $\mathrm{pH}$ of the same solution back to 7, the DLS diameters of the nanogels in the presence of the IONPs was $191 \pm 8 \mathrm{~nm}$, as opposed to the DLS diameter equal to $137 \pm 29 \mathrm{~nm}$ for the empty nanogels (Table 1, Supporting Information). TEM characterization confirmed the presence of IONPs within the nanogel structure (Figure 3C). The nanogels loaded with IONPs could release their payload by switching the $\mathrm{pH}$ again from 7 to 3.5. Indeed, after $3 \mathrm{~h}$ at $\mathrm{pH} 3.5$, most of the particles were released from nanogels, as confirmed by TEM (Figure 4).

In order to rationalize and understand the driving force for the loading, we have characterized the system in more detail by analyzing the surface charge of the individual units, namely, the nanogels and the IONPs, and that of the nanogels loaded with IONPs at various $\mathrm{pH}$ values. At $\mathrm{pH} 3.5$, the surface charges of the swollen nanogels and those of the IONPs were both positive (zeta potentials were $+56 \mathrm{mV}$ and $+8 \mathrm{mV}$, respectively), and at the same time, the nanogels were in the swollen state, which promoted the entrapment of the nanoparticles at their interior. We have also attempted to load the nanogels at $\mathrm{pH} 7$ instead of $\mathrm{pH}$ 3.5. At this $\mathrm{pH}$, the surface charge of the nanogels was still moderately positive, while that of the IONPs was negative. The negative charge is likely due to the charge balance at the surface of IONPs given by the sum of amino-PEG moieties and carboxyl-terminated groups of the polymer (zeta potentials are +28 and $-42 \mathrm{mV}$, respectively; see Table 2, Supporting Information). Therefore, even if at this $\mathrm{pH}$ value the nanogels were swollen, one should expect a higher electrostatic interaction between the nanogels and the IONPs. We observed indeed that also after incubation under these conditions we could load IONPs within the nanogels (Figure 4S, Supporting Information). A loading experiment was attempted even at $\mathrm{pH} 10$, at which the surface charge of the nanogel was only slightly positive (zeta potentials for the nanogels and for the IONPs were +15 and $-42 \mathrm{mV}$ ); hence, the electrostatic interactions between the nanogels and the IONPs were weaker than at $\mathrm{pH}$ 7. In this case, we could still observe (by TEM) the adsorption of a few nanoparticles on the surface of the nanogels, but most nanogels had not been able to incorporate the IONPs (Figure 4S, Supporting Information).

For the quantification of IONPs loaded within the nanogel at the different $\mathrm{pH}$ values, the various samples were digested in $\mathrm{HCl} / \mathrm{HNO}_{3}$, and their iron content was estimated by means of elemental analysis, which allowed us to estimate quantitatively 


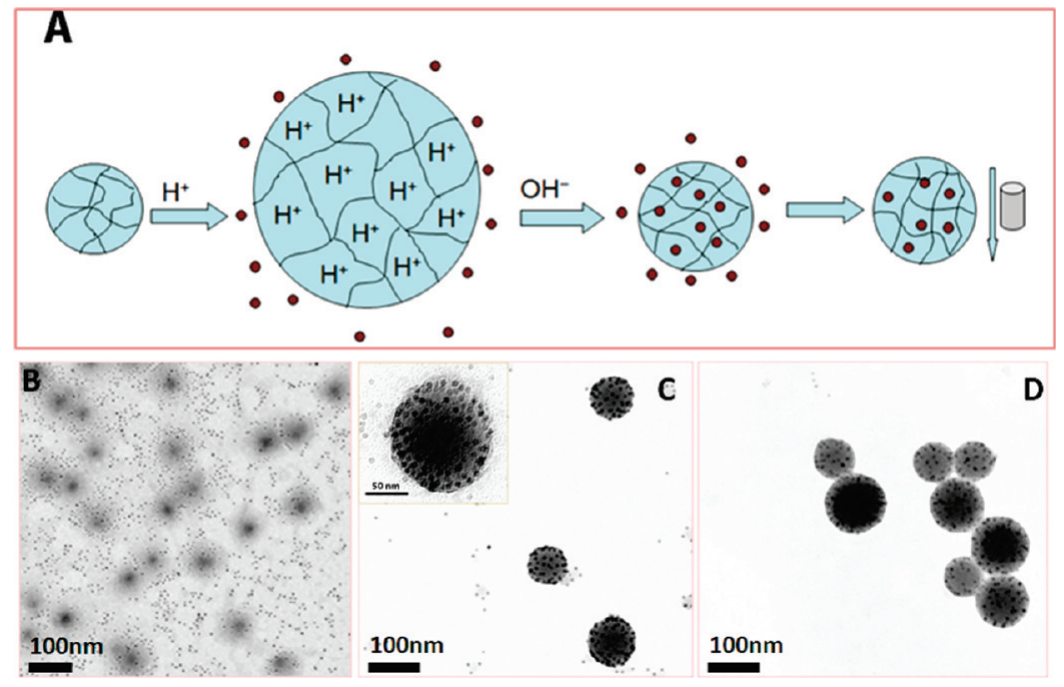

Figure 3. (A) Scheme of the loading of magnetic nanoparticles within the $\mathrm{pH}$-responsive nanogels. Corresponding TEM characterization of the different steps: (B) at acidic $\mathrm{pH}$ the nanogels were mixed with the IONPs; (C) after $12 \mathrm{~h}$, the $\mathrm{pH}$ was switched back to $\mathrm{pH} 7$, such that the IONPs were entrapped within the nanogel network. (D) The application of a small magnet helped to remove most of the free IONPs in solution. A complete cleaning was achieved by performing an additional purification step on a Sephadex column.

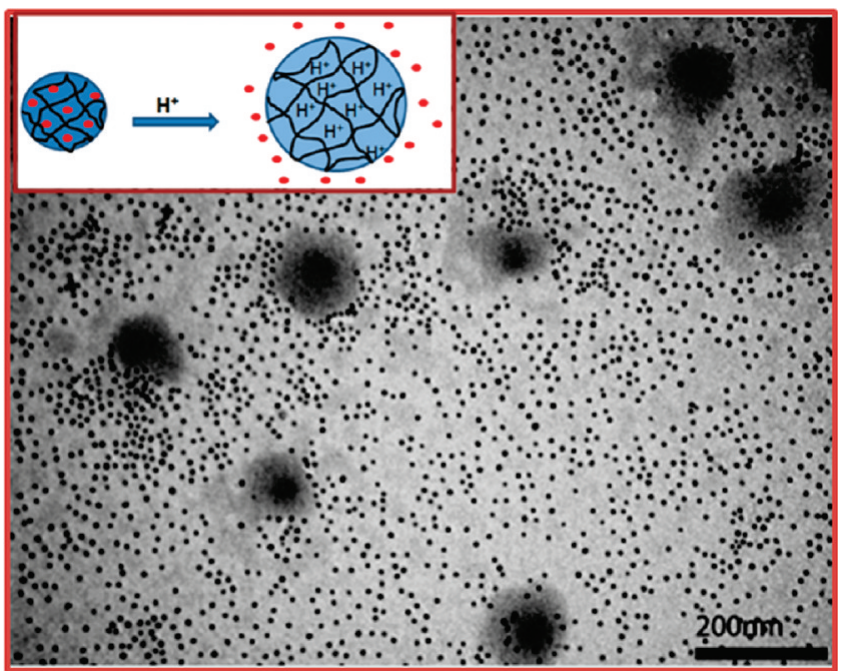

Figure 4. Release experiment of the IONPs. Switching the $\mathrm{pH}$ of the IONP-loaded nanogel solution from 7 to 3.5 induced the swelling of the nanogels and consequently the release of the IONPs entrapped within the polymer network.

the number of IONPs entrapped within the nanogels. ${ }^{35}$ The highest concentration of IONPs was found in the beads loaded at $\mathrm{pH} 3.5$, followed by those loaded at $\mathrm{pH} 7$ and then by those at pH 10 (see Table 2S, Supporting Information). Those results were reproducible and provided a clear indication of the average loading efficiency of the nanogels. These data nicely correlate with the trend in IONP-loaded nanogel diameter (as estimated by the DLS), which is bigger for the gels loaded at $\mathrm{pH} 3.5$, followed again by those loaded at $\mathrm{pH} 7$ and at $\mathrm{pH} 10$, respectively. This correlation suggests that the swelling behavior is the main driver for the encapsulation of IONPs, although a contribution due to electrostatic interactions between the nanogels and the IONP surface cannot be excluded.

Once the nanogels were loaded with IONPs, their surface charge became negative at $\mathrm{pH}$ 7. However, the trend in absolute values of surface charges was reversed in this case: it was higher for the nanogels loaded at $\mathrm{pH} 10$, followed by those loaded at $\mathrm{pH}$ 7 and $\mathrm{pH} 3.5$, respectively. This might likely be attributed to a much lower fraction of nanoparticles adsorbed at the nanogel surface with respect to those trapped deeper in the network structure of the nanogel at $\mathrm{pH} 3.5$ (the nanoparticles contributed with negatively charges). ${ }^{31,33}$

3.3. TEM Characterization of the Entrapment of the IONPs within the Nanogels. In order to confirm the entrapment of the IONPs, we carried out additional TEM characterization. Several bright field electron microscopy (BF-EM) images of a nanogel sample loaded with IONPs were taken at different tilts on a large angular range (from $-55^{\circ}$ to $0^{\circ}$, to $60^{\circ}$ ). Two BF-EM images of the same IONP loaded nanogel are shown in Figure 5A, B. Figure $5 \mathrm{~A}$ corresponds to the specimen tilted at $0^{\circ}$ (i.e., the plane of the sample is basically normal to the electron beam direction) and Figure 5B corresponds to the same sample tilted by $60^{\circ}$. From the high-tilt image, two main considerations can be made: first, the projection of the light gray zone (observed circular at $0^{\circ}$ tilt) is elliptical at high tilt, indicating that the polymer behaves as a sphere pressed on the plane of the carbon grid in a direction perpendicular to it. Second, at high tilt the spherical nanoparticles are located inside the contours of the light-gray zone that corresponds to the polymer. This suggests that the IONPs were embedded within the first few polymer layers. If, on the other hand, the IONPs were simply attached on the surface of the polymeric crushed sphere, they should have appeared also on the external side of the light-gray zone's contour.

In order to localize the radial distribution of IONPs within the nanogels, we have performed TEM on the cross sections of the IONP-loaded nanogels, which had been embedded within a paraffin resin. The sections analyzed had thickness of $70 \mathrm{~nm}$ (Figure 5C) and $50 \mathrm{~nm}$ (inset of Figure 5C), respectively. As observed in Figure 5C, most of the IONPs were densely packed at the edge of the beads, within the first layers of the polymer, and only few of them were found deep inside in the nanogels. It is interesting to compare these results with the cross-sectional images of the same type of nanogel used as template for the gold synthesis reported by Nakamura. ${ }^{38}$ In that case, as the gold nucleation occurred only at the surface on the TEM cross sections, no nanoparticles were found within the nanogel.

(38) Kensuke Akamatsu, , Takaaki Tsuruoka, Hidemi Nawafune, Syuji Fujii Yoshinobu Nakamura Langmuir 2009, [Online early access]. 

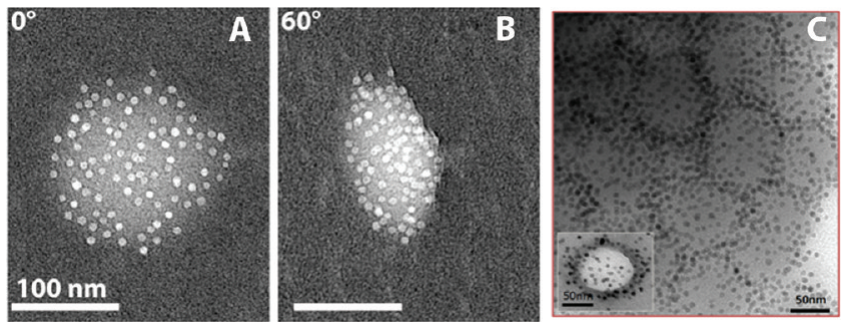

Figure 5. (A,B) Inverted bright field electron microscopy images of a sample of nanogel loaded with IONPs. (A) corresponds to the specimen tilted by $0^{\circ}$ and (B) corresponds to the same sample tilted by $60^{\circ}$ under the electron beam. On the tilted sample, the light gray edge, corresponding to the polymer, covers the bright spots, which are the magnetic nanoparticles. (C) Cross-sectional TEM image of nanogel loaded with IONPs recorded on a section having a thickness of $70 \mathrm{~nm}$ (while for the inset the section thickness is of $50 \mathrm{~nm}$ ).

It is also worth noting that our IONP-loaded nanogels can be kept for months at $\mathrm{pH} 7$ and at room temperature without observing leakage of IONPs. This is likely an indication of the entrapment of the particles within the polymer networks. Taken all together, these results point to the entrapment and localization of the IONPs within the first layers of the polymer network in the nanogel. This configuration rationalizes the loading and thus the consequent release of the IONPs that we observe. Our results are in agreement with those reported by Jang et al. who used hydrogel spheres based on a thermoresponsive polymer PNIPAM and $\mathrm{pH}-$ responsive units 4-vinylpyridine to entrap CdTe nanoparticles. In that case, however, the authors provided other indirect proofs that pointed to the nanoparticle entrapment. ${ }^{39}$

Additionally, it is also worth noticing that, if the IONPs are left at $\mathrm{pH} 3.5$ overnight, no change in the composition or shape of the nanoparticles was observed, and no leakage of $\mathrm{Fe}^{3+}$ was detected in the acidic medium.

On the basis of the above results, we decided in all the subsequent experiments to load the IONPs at pH 3.5, as in these conditions we achieved the highest efficiency of nanoparticle loading.

3.4. Loading and Release Experiments of Oligonucleotides. We have applied the procedure described above to load and release short oligonucleotide sequences of about 25 bases. In order to detect the loading and release process, we have employed a sequence bearing at the $5^{\prime}$ end the fluorophore molecule $\mathrm{Cy} 3$, which allowed us to track the presence of the Cy3-DNA within the nanogel by photoluminescence (PL) spectroscopy (Figure 6) and UV-visible absorption spectroscopy (Figure 5S, Supporting Information). Figure 6 shows the PL spectra of free Cy3-DNA (red line) and of the nanogels loaded with Cy3-DNA (black line), after the solution was purified from the excess Cy3-DNA (see section 1.5 of the Supporting Information). When loaded in the gels, the Cy3-DNA exhibited a PL spectrum that was red-shifted by about $3 \mathrm{~nm}$ with respect to that of free Cy3-DNA.

The signal recorded was due only to the Cy3-DNA loaded into the nanogel and not to free Cy3-DNA. As proof, we have recorded the PL spectra after each washing step (the solution recovered from the lower part of the centricone filter used for the purification). The signal of free Cy3-DNA in this solution was progressively reduced, and after 6 washing steps there was no PL signal from free Cy3-DNA. These data clearly confirmed the loading of the Cy3-DNA within the nanogel. The Cy3-DNA loading was further corroborated by DLS measurements, since an increase in the

(39) Kuang, M.; Wang, D. Y.; Bao, H. B.; Gao, M. Y.; Mohwald, H.; Jiang, M Adv. Mater. 2005, 17, 267.

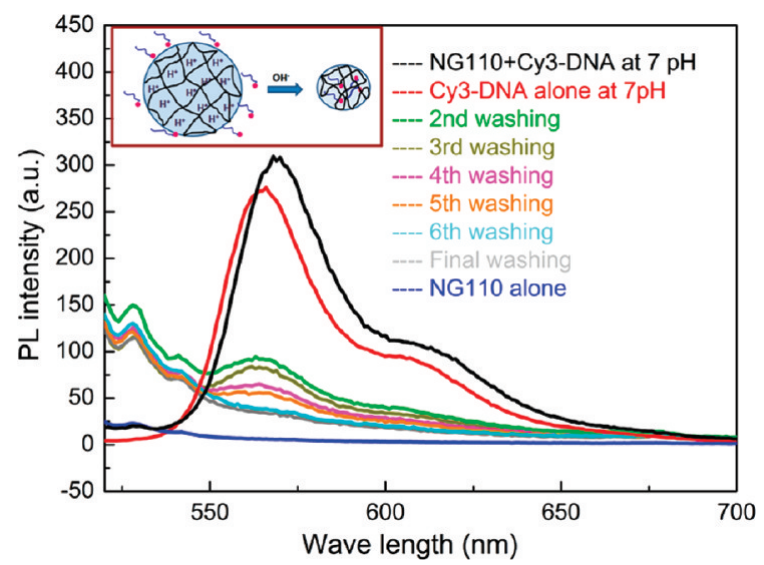

Figure 6. PL spectrum of Cy3-DNA loaded within the nanogel after the cleaning procedure had been applied (black curve); PL spectrum of the free Cy3-DNA (red curve) and starting nanogel solution (blue curve). The plot reports in addition the PL spectra of aliquots collected at the different washing steps, as well as those of the loaded nanogel solution, Cy3-DNA and nanogel only. After 6 washing steps, the free Cy3-DNA was removed completely from the solution containing the loaded nanogels. The inset shows a scheme of the loading of Cy3-DNA within the nanogel.

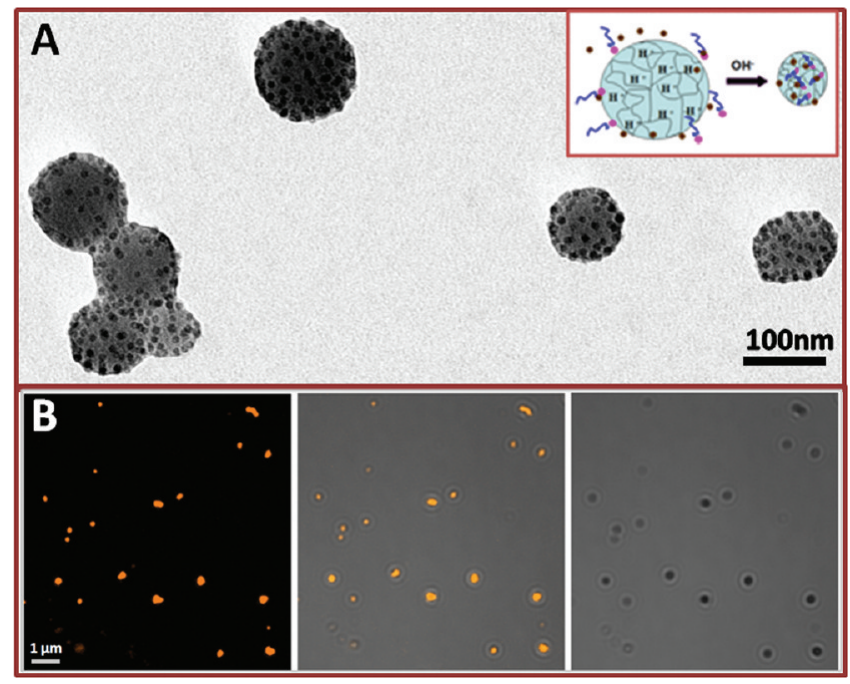

Figure 7. (A) TEM characterization of nanogels loaded simultaneously with IONPs and Cy3-DNA. (B) Confocal microscopy characterization of the sample shown in A. The fluorescent signal of the DNA bearing the $\mathrm{Cy} 3$ marker (left panel) is colocalized with the spots seen in the phase contrast image of the nanogel (right panel). The central panel is a merged image of both panels.

average nanogel hydrodynamic diameter was observed after loading (i.e., from $137 \pm 29 \mathrm{~nm}$ to $165 \pm 63 \mathrm{~nm}$ for a nanogel sample loaded at $\mathrm{pH} 3.5$ and measured at $\mathrm{pH} 7$ (the dye signal is quenched at $\mathrm{pH}$ 3.5); see Table 1S, Supporting Information).

3.5. Simultaneous Loading and Release of Oligonucleotides and IONPs. In a third series of experiments, we have loaded simultaneously IONPs and the oligonucleotide sequences in the nanogels, by mixing together solutions of IONPs, Cy3DNA, and nanogels, according to the protocols described above. The simultaneous loading of IONPs and Cy3-DNA was confirmed by a combination of TEM measurements, by which we could locate the IONPs in the nanogels, and by confocal microscopy, by which we could identify the PL signal from the Cy3DNA within the nanogel (Figure 7). 


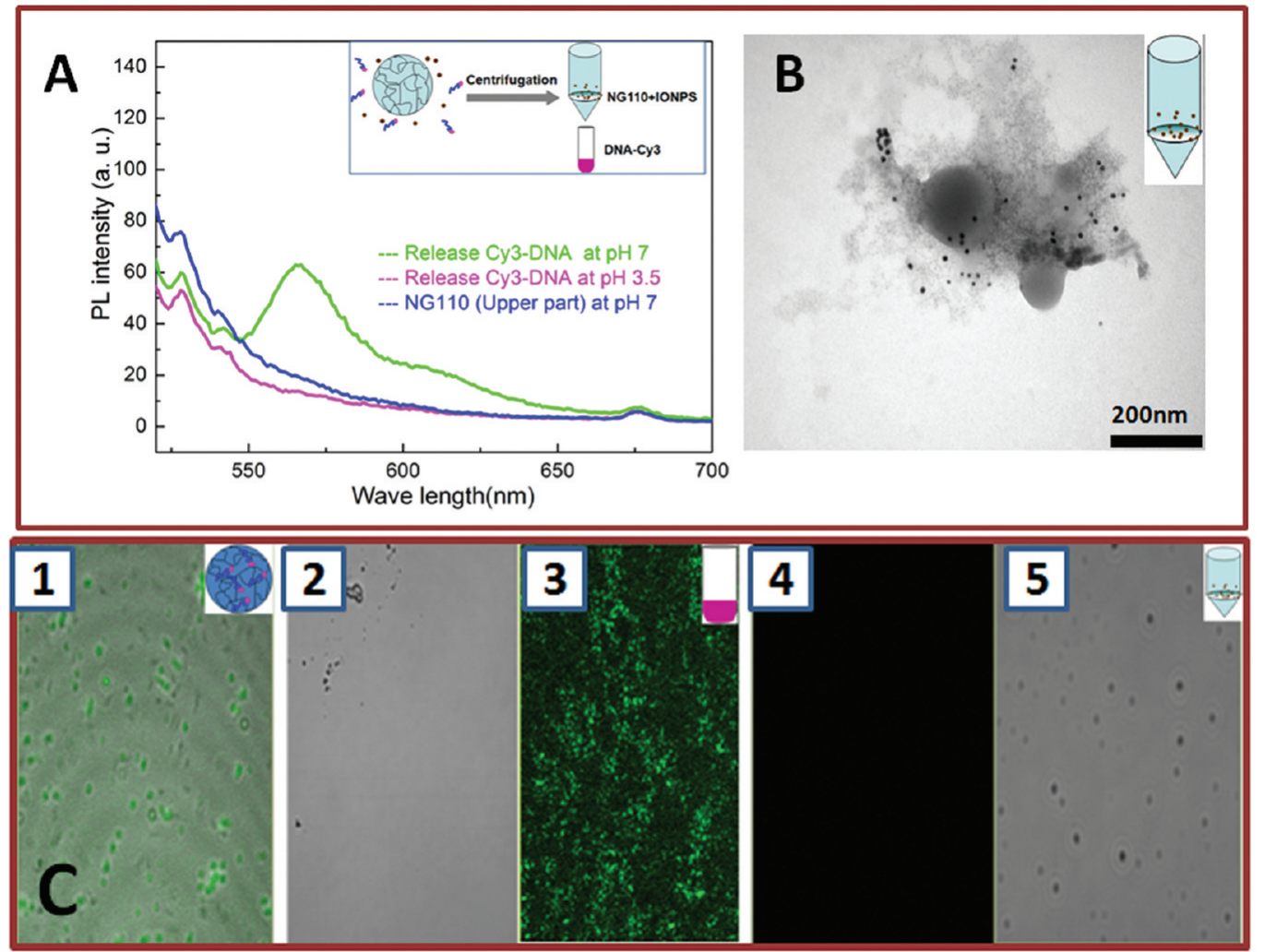

Figure 8. (A) PL characterization of the release process. The inset is a sketch showing the separation on the centrifuge filter between the IONPs and nanogels from the small Cy3-DNA molecules. The green curve corresponds to the Cy3-DNA signal recorded on the lower side of the centrifugation filter after $72 \mathrm{~h}$ at $\mathrm{pH} 7$, while the blue curve corresponds to the PL signal recorded on the upper side of the filter. The violet curve corresponds to the PL of Cy3-DNA recovered on the lower side of the centrifuge filter (at $\mathrm{pH} 3.5$, the dye signal is quenched). (B) Corresponding TEM characterization of the sample recovered on the upper side of the membrane. The IONPs released by the nanogels are retained on the upper side of the filter. In addition, the nanogel structure appears damaged after the simultaneous release of both cargo elements. (C) Confocal characterization of the release process: the image shown in $\mathrm{C} 1$ is obtained by merging the fluorescent and the phase contrast images of the same area of the nanogels loaded with IONPs and Cy3-DNA, before the release. C2 and C3 correspond, respectively, to the phase contrast and fluorescent signal of Cy3-DNA released by the nanogel and collected at the bottom side of the filter. C4 and C5 correspond, respectively, to the fluorescent and phase contrast images of the same areas of the sample collected on the upper side of the filter at $\mathrm{pH}$ 7. After the release, while the nanogels were still visible (C5), no signal was recorded in the corresponding fluorescent channel (C4)), indicating the completed release of DNA by the nanogels.

Despite the confocal images were taken by working at the resolution limit of the confocal setup (hence the nanogel particles could not be focalized), on dilute solutions these fluorescent spots were colocalized with spots in the corresponding phase contrast images, and which could be ascribed to the nanogels (Figure 7B).

Additionally, under the same experimental conditions the TEM and DLS diameters of the nanogels simultaneously loaded with IONPs and Cy3-DNA were bigger than those of the corresponding nanogels loaded either with Cy3-DNA or with IONPs alone (Supporting Information Table 1S). As an example, the diameter of the loaded nanogel increased to $250 \pm 50 \mathrm{~nm}$ (by DLS) and the zeta potential became negative $(-10.5 \pm 1.5 \mathrm{mV})$. Additional PL characterization of the nanogels loaded with Cy3DNA and IONPs was performed and confirmed the presence of DNA (Supporting Information Figure 6S).

In order to release the multicargo, the nanogels were first equilibrated at $\mathrm{pH} 3.5$ in a solution containing $140 \mathrm{mM} \mathrm{NaCl}$. To achieve complete release of the DNA from the nanogel, it was necessary to keep the nanogel at $\mathrm{pH} 3.5$ for $72 \mathrm{~h}$. After this time, we first separated the Cy3-DNA from the nanogel and IONP portions by using centrifuge filters. By choosing an appropriate pore size for the membrane, we could retain the IONPs and the nanogel on the upper side of the membrane, while molecules like Cy3-DNA (see inset Figure 8A) were able to pass through the membrane. By recording the PL spectra on the fraction collected at the bottom side of the membrane, we could verify the release of the oligonucleotides (Figure 8A). It is worth noting that after $24 \mathrm{~h}$ we could still record the fluorescent signal, not only on the lower part of the centrifuge tube, but also on the upper part of the membrane (data not shown). Only after $72 \mathrm{~h}$ was a complete release of the DNA achieved, since at this time no further PL signal was detected on the upper side of the membrane.

These data were also supported by confocal microscopy observations on the various aliquots that had been recovered from each side of the filter (Figure 8C). When both Cy3-DNA and the IONPs were packed within the nanogels, in the confocal fluorescence image the spots appeared point-like. In addition, they were colocalized with spots in the phase contrast image (Figure 8C1). After the complete release, on the upper side of the filter it was still possible to capture the phase contrast image of the nanogel, while no fluorescence could be recorded in the corresponding channel (Figure 8C4 and $\mathrm{C} 5$ ). The portion recovered from the lower part of the membrane still showed a fluorescent signal. This signal, however, was not clumped any more in pointlike regions, but was rather distributed homogeneously in the whole field of view. This could be interpreted as arising from the Cy3-DNA that had been released from the nanogels (Figure 8C3). In the corresponding phase contrast image, the nanogels could not be seen. TEM analysis of the part retained on the upper side of 
the filter indicated the presence of both released IONPs and nanogels, but the nanogels appeared disrupted in this case (Figure 8B). These findings are somehow unique, since in all previous experiments involving either DNA or IONPs, unloading the nanogels had retained their original shape. Apparently, the simultaneous release of both DNA and IONPs was responsible for this effect.

Such irreversible swelling during unloading of both DNA and IONPs deserved a deeper analysis. We tested therefore the effect of the $\mathrm{pH}$ on the swelling of nanogels (both with and without the cargo) by switching the $\mathrm{pH}$ of the medium from 8 to 3 and back. Swelling of the empty nanogels was reversible, since the curve describing their size dependence on the $\mathrm{pH}$, when this was cycled from 3.5 to 8 and back, did not show any hysteresis (Figure 7S A, Supporting Information). These results are in agreement with previously published data. ${ }^{36} \mathrm{~A}$ similar behavior was also observed in the case of nanogels loaded with IONPs (Figure 7S C, Supporting Information), while an appreciable hysteresis was recorded on the nanogels loaded with Cy3-DNA (Figure 7S B, Supporting Information).

The situation was drastically different when the nanogels were filled with both IONPs and Cy3-DNA (Figure 7S D, Supporting Information). This time the curve describing the size dependence on the $\mathrm{pH}$, when this was increased from 3.5 to 8 (the "forward curve"), did not overlap with the corresponding curve when the $\mathrm{pH}$ was decreased from 8 back to 3.5 (the "backward curve"). Starting from $\mathrm{pH}$ 6, the nanogel size from the backward curve was always higher than that from the forward curve, pointing to a modification in the structure of the nanogel after it was used as cargo. These data, together with the TEM characterization, are indicative of the structural degradation of the nanogel after the simultaneous release of Cy3-DNA and IONPs (Figure 8).

For the quantification of DNA loaded within the nanogel, calibration curves of PL/[DNA] (photoluminescence/DNA concentration) were used (Figure 8S, Supporting Information). Using those curves, we found that, when only DNA was loaded within the nanogel, the amount of DNA that could be actually loaded corresponded to about $16 \%$ of the initial DNA added (which corresponded to an amount of DNA equal to $0.048 \mathrm{pmol} /$ $\mu \mathrm{L}$ for $0.053 \mathrm{~g}$ weight of nanogels). On the other hand, the amount of DNA loaded in the case of simultaneously loading of DNA and IONPs was slightly higher and corresponded to about $20 \%$ of the initial DNA added $(0.0623 \mathrm{pmol} / \mu \mathrm{L}$ of DNA for $0.053 \mathrm{~g}$ weight of nanogels).

3.6. Salt Effect on the Swelling of the Loaded Nanogels. The swelling behavior of the nanogel was affected by the presence of salt in solution (Figure 9). We report here only the data related to nanogels loaded with IONPs. At $\mathrm{pH} 8$, for instance, the size of the loaded nanogels was not altered significantly by the presence of salt. At $\mathrm{pH} 7.4$, on the other hand, the loaded nanogels in $100 \mathrm{mM}$ and $200 \mathrm{mM} \mathrm{NaCl}$ solutions were bigger than those loaded in plain water, by about 50 and $70 \mathrm{~nm}$, respectively. At pH 6.5, the loaded nanogels in $100 \mathrm{mM}$ and $200 \mathrm{mM}$ $\mathrm{NaCl}$ solutions were affected significantly by the presence of salt in solution, since an increase in size of 130 and $150 \mathrm{~nm}$, respectively, as compared to the sample of nanogel in water was recorded.

At $\mathrm{pH} 3.9$, the differences in size were even more remarkable: the nanogels loaded with IONP in $200 \mathrm{mM} \mathrm{NaCl}$ were again the biggest (their diameter was around $850 \mathrm{~nm}$, which corresponded

(40) Fujii, S.; Dupin, D.; Araki, T.; Armes, S. P.; Ade, H. Langmuir 2009, 25 , 2588 .

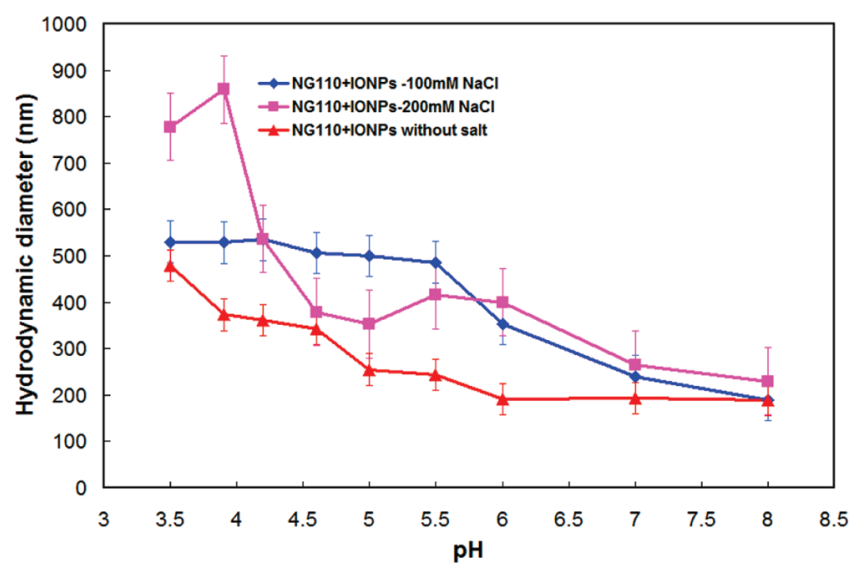

Figure 9. Salt effect on the swelling behavior of nanogels loaded with IONPs in plain water (red curve), $100 \mathrm{mM} \mathrm{NaCl}$ (blue curve), and $200 \mathrm{mM} \mathrm{NaCl}$ (violet curve).

almost to their swelling limit), followed by those loaded at $100 \mathrm{mM}$ $(529 \mathrm{~nm})$, and was still $373 \mathrm{~nm}$ for the nanogel in water.

It has been reported by others ${ }^{40}$ that on the vinyl pyridinedivinyl benzene-based nanogels, the addition of salts reduces the screening effect of the charges, resulting in a reduced swelling of the nanogels. The higher the amount of salt added, the stronger the screening effect is, and thus the lower the extent of swelling of the nanogel. Also, in our case, when the nanogels were loaded with IONPs, the swelling behavior in the presence of salt had a trend in the $\mathrm{pH}$ range from 7 to 4.2 that was similar to that of previous reports. Namely, the addition of $200 \mathrm{mM} \mathrm{NaCl}$ resulted in a reduced swelling with respect to $100 \mathrm{mM}$. The difference occurred for the swelling of the IONP-loaded nanogel below 4.2 in $200 \mathrm{mM}$, which is bigger than that in $100 \mathrm{mM}$. The IONPs have charged groups at their surface, and those groups can coordinate counterions in their surroundings. This results in a high local ionic strength that can break the nanogel structure and consequently increase the DLS diameter of the polymer. This behavior has been confirmed by TEM images of the nanogel under these conditions (data not shown).

The different diameters of the nanogels in a solution $100 \mathrm{mM}$ $\mathrm{NaCl}$ indicate that the leaking of the IONPs occurred already at $\mathrm{pH}$ close to 6.5 (which is actually the $\mathrm{pH}$ of the extracellular tumor environment), while no appreciable leakage was observed at pH 7.4 (which is the pH of the blood) (Figure 9).

\section{Conclusions}

In this work, we have reported the fabrication of a multivalent nanosystem based on a class of functional molecules known as stimuli responsive polymers, which can work as cargo system for gene (or drug) delivery, and which can entrap at the same time inorganic magnetic nanoparticles. Differently from previously reported studies, the magnetic nanoparticles in this work are not covalently linked to the gel networks, and thus they can be loaded and released by tuning the $\mathrm{pH}$. The full characterization provided when only DNA or IONPs or a combination of them were employed has allowed us to understand both the mechanism by which the different payloads are retained within the gel and the release process as a function of the $\mathrm{pH}$ and of the salt concentration.

The system developed in this work, especially in the case when both DNA and IONPs have been loaded, has interesting features and might find application as a therapeutic agent. It can act as a heat mediator for performing hyperthermia, as gene delivery system (for instance in si-RNA treatment), and at the same time 
as an imaging contrast agent in MRI. The magnetic nanocarriers developed appear to have the right geometry for performing those tasks. Preliminary studies by other groups ${ }^{41-43}$ have shown indeed that clustering of IONPs (like in our case when they are loaded in the nanogels) improves the relaxivity signals recorded with respect to individual magnetic nanoparticles. On the other hand, hyperthermia seems more efficient when the magnetic nanoparticles are not encapsulated within a matrix, but they are freely delivered to a certain target site. ${ }^{29}$ Our system appears to have the right features for such purposes. When circulating in a medium with $\mathrm{pH}$ below 7.4, like blood, the nanogels will be in a packed configuration, allowing for a better enhancement of the MRI signal form the IONPs. On the other hand, once the nanogels will be delivered to a compartment with a $\mathrm{pH}$ below 6.5 , like the extracellular tumor environment, they would begin to swell, and thus they would release the IONPs. The hyperthermia treatment could be therefore performed on the IONPs, once they will be delivered into the extracellular tumor environment, where the $\mathrm{pH}$ is around 6.5. The further uptake by tumor cells would allow the system to experience the different $\mathrm{pH}$ of the intracellular compartments. For such a purpose, cellular studies are now under investigation in our laboratory.

(41) Nasongkla, N · Bey, E; Ren, J M · Ai, H : Khemtong, C : Guthi, J. S ; Chin, S. F.; Sherry, A. D.; Boothman, D. A.; Gao, J. M. Nano Lett. 2006, 6, 2427.

(42) Taboada, E.; Solanas, R.; Rodriguez, E.; Weissleder, R.; Roig, A. Adv. Funct. Mater. 2009, 19, 2319.

(43) Fresnais, J.; Berret, J. F.; Petesic, B. F.; Sandre, O.; Perzynski, R. Adv. Mater. 2008, 20, 1 .
Additionally, it is worth noting that the nanogels developed by us could also work as a targeting tool to deliver the payload with a spatial and temporal control. The presence of magnetic nanoparticles allows spatially controlled delivery, since the nanogels feel an externally applied magnetic field and thus might be attracted to specific locations of the body, where the magnetic field will be placed. Temporally controlled delivery will be ensured by the variations in $\mathrm{pH}$ that the nanogels will sequentially experience during their journey (hence by the response of the nanogels to these variations) in the various body/cellular compartments. The synergy between both effects might allow a more efficient delivery of the nanogel cargos.

Acknowledgment. This work was supported in part by the European project Magnifyco (Contract NMP4-SL-2009-228622). We thank Mario Malerba for TEM sample preparation and Sergio Marras for helpful discussion.

Supporting Information Available: Experimental details; two tables summarizing the DLS and TEM diameters and the zeta potential measurements; additional TEM characterization images of the nanogels, additional PL and absorption measurements of the nanocarriers and swelling behavior of different loaded nanogel solution in the decreasing and increasing $\mathrm{pH}$ profiles; PL/[Cy3-DNA] calibration curves. This material is available free of charge via the Internet at http://pubs.acs.org. 